CERN-TH.6662/92

\title{
More About Discrete Gauge Anomalies
}

\author{
Luis E. Ibáñez * \\ CERN, 1211 Geneva 23, Switzerland
}

\begin{abstract}
I discuss and extend several results concerning the cancellation of discrete gauge anomalies. I show how heavy fermions do not decouple in the presence of discrete gauge anomalies. As a consequence, in general, cancellation of discrete gauge anomalies cannot be described merely in terms of low energy operators involving only the light fermions. I also discuss cancellation of discrete gauge anomalies through a discrete version of the Green-Schwarz (GS) mechanism as well as the possibility of discrete gauge R-symmetries and their anomalies. Finally, some phenomenological applications are discussed. This includes symmetries guaranteeing absence of FCNC in two-Higgs models and generalized matter parities stabilizing the proton in the supersymmetric standard model. In the presence of a discrete GS mechanism or/and gauge R-symmetries, new possibilities for anomaly free such symmetries are found.
\end{abstract}

* Adress after September 1992: Departamento de Fisica Teórica C-XI, Universidad Autónoma de Madrid, Cantoblanco, 28049, Madrid, Spain. 


\section{Introduction}

Discrete symmetries are often impossed in Lagrangian field theories for a variety of phenomenological motivations. Such kind of symmetries seem to be required e.g. in different extensions of the standard model like multi-Higgs or supersymmetric models in order to avoid large flavour-changing neutral currents or too fast proton decay. There is nothing wrong with discrete symmetries except for the apparent lack of fundamental (non-phenomenological) motivation for their existence and the arbitrary number of existing possibilities. A further problem for discrete symmetries may appear if indeed, as argued by some authors, gravitational (e.g. wormhole [1]) corrections badly violate all non-gauge symmetries.

Those problems could be solved if the physically relevant discrete symmetries were 'gauge' discrete symmetries [2], [3], [4]. Such discrete gauge symmetries have been argued to be stable against large gravitational corrections [2],[3]. Furthermore it was pointed out in ref. [5] that discrete gauge symmetries are restricted by certain anomaly cancellation conditions. Many candidate gauge discrete symmetries may be ruled out on the basis of these conditions. This has been applied in particular to the discrete symmetries ensuring proton stability in the supersymmetric standard model (SSM) [6], solar neutrino models [7], [8] and other phenomenological questions [9], [10]. The possibility of interpreting CP as a gauge discrete symmetry has also been considered in ref. [11].

In the present paper I discuss further several aspects of the cancellation of discrete gauge anomalies and clarify several issues raised in refs. [5], [6] and [12]. In section two I emphasize a peculiar property of the discrete gauge anomaly cancellation conditions which is that, in general, heavy fermions do not decouple in the presence of discrete gauge anomalies. This is reminiscent of the work of ref. [13]in which it was shown that the top-quark does not decouple if one tries to make it infinitely heavy in the standard model. This is particularly the case of the cubic $Z_{N}$ discrete gauge anomaly and the mixed $Z_{N}$-gravitational anomaly which are sensitive to massive fermions. In the case of the mixed $Z_{N}-S U(M)$ anomalies, the contribution of light and heavy fermions to the anomaly cancels separately and hence the discrete anomaly is not sensitive to heavy fermions. 
That is why the $Z_{N}-S U(M)$ anomaly may be understood just in terms of the relevant 't Hooft fermionic operator involving only the massless fields, a point of view which has specially been emphasized in ref.[12]. On the other hand, the cubic $Z_{N}$ and the mixed $Z_{N}$-gravitational anomalies are not easy to understand from that point of view. Nevertheless, even though these two anomalies depend on the heavy sector of the theory, they do it in a definite manner which make possible to obtain usefull constraints from them.

In section three I briefly discuss the cancellation of discrete anomalies for the case of 'gauge' R-symmetries. This also provides us with an specific example of the problems one may get if one tries to interpret all discrete anomalies in terms of a 't Hooft fermionic operator. The presence of discrete gauge anomalies in four-dimensional strings is discussed in section four. I also present several examples and describe the way in which a discrete version of the Green-Schwarz mechanism operates [6],[12](see also [14]). In section five I consider a couple of phenomenological applications including symmetries supressing flavour changing neutral currents in two-Higgs models and symmetries supressing fast proton decay in the supersymmetric standard model. In particular, I discuss how the analysis in ref.[6] is modified if one considers i) discrete R-symmetries and/or ii) a discrete Green-Schwarz mechanism. I find that in such a case new 'Generalized Matter Parities' could be anomaly free. However, the presence either of gauged R-symmetries and/or a discrete (GS) mechanism are only expected in theories with higher dimensions like strings. Some final comments and comparison with other approaches [15] are left for the sixth section.

\section{The Discrete Gauge Symmetry Anomaly Cancelation Conditions and the Non-Decoupling of Heavy Fermions}

Let us start by briefly recalling the discrete gauge anomaly conditions derived in ref.[5]. Let us assume there is an effective gauge $Z_{N}$ symmetry under which the massless fermions of the theory transform with charges $q_{j}$. There are several types of "discrete anomalies" which must be cancelled by appropriately choosing the chiral fermion content of the theory. These are the following: 
i) Cubic $Z_{N}^{3}$ anomaly cancelation condition:

$$
\sum_{i}\left(q_{i}\right)^{3}=r N+\eta s \frac{N^{3}}{8}, s \in \mathbf{Z} .
$$

where $\eta=1,0$ for $N=$ even, odd.

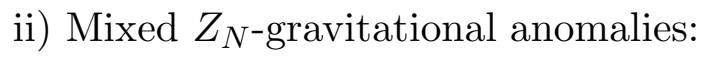

$$
\sum_{i}\left(q_{i}\right)=r^{\prime} N+\eta n^{\prime} \frac{N}{2}, n^{\prime}, r^{\prime} \in \mathbf{Z} .
$$

iii) Mixed $Z_{N^{-}} S U(M)-S U(M)$ anomalies:

$$
\sum_{i} T_{i}\left(q_{i}\right)=\frac{1}{2} r^{\prime \prime} N \quad, r^{\prime \prime} \in \mathbf{Z} .
$$

Here $T_{j}$ is the quadratic $S U(M)$ Casimir corresponding to each given representation (the normalization is such that the Casimir of an $M$-plet is $=1 / 2$ ). The sums are over the light sector of the theory (those fields which do not get a mass when the symmetry breaking $U(1) \rightarrow Z_{N}$ takes place).

If there are extra unbroken abelian factors, $U(1)_{u} \mathrm{~s}$, in the theory one can write down further constraints associated with mixed $U(1)_{u}^{2} Z_{N}$ and $U(1)_{u} Z_{N}^{2}$ anomalies. However [5] they turn out not to be very usefull without the precise knowledge of the underlying theory before the symmetry breaking $U(1) \rightarrow Z_{N}$ and, particularly, the normalization of the $U(1)$ 's. If that normalization is known (e.g., if it is known that those $U(1)$ 's were embedded in some simple group), these anomalies are restrictive of the massless fermion content and should be taken into account.

Let us discuss some interesting aspects of the above anomaly conditions.

i) In the cubic $Z_{N}^{3}$ anomaly conditions (1) above we have not indicated whether $r$ should be taken an integer or not. In fact, it will always be an integer if the massive fermions which gained masses in the process $U(1) \rightarrow Z_{N}$ have 
integer $Z_{N}$ charges. It is easy to see [5] that the contribution of massive fermions $\Psi_{1}^{j}, \Psi_{2}^{j}$ with charges $Q_{1}^{j}, Q_{2}^{j}$ to the coefficient $r$ in (1) is given by

$$
r_{\Psi}=-\sum_{j=\text { heavies }} p_{j}\left(3 Q_{1}^{j^{2}}-3 p_{j} N Q_{1}^{j}+p_{j}^{2} N^{2}\right)
$$

where the $p_{j}$ are integers obeying $Q_{1}^{j}+Q_{2}^{j}=p_{j} N$. These integers $p_{j}$ carry information about the charges of the scalar fields which break the symmetry $U(1) \rightarrow Z_{N}$. In (1) one has $r=r_{\Psi}+$ integer. It is then obvious that, if the massive fermions all have integer charges, $r$ will be an integer ${ }^{*}$. In the absence of specific information about the massive sector, one cannot be sure about the nature of $r$ which may perfectly be a fractional number. In this case the cubic anomaly is not very usefull in constraining low-energy physics [12],[6] . On the other hand one can reverse things and constraint the massive sector from anomaly cancellation [12],[6]. Indeed, since by definition $\sum_{i} q_{i}^{3}$ is an integer, $N \times r_{\Psi}$ has to be an integer which yields the consistency condition

$$
3 N \sum_{j=\text { heavies }} p_{j} Q_{1}^{j} Q_{2}^{j}=\text { integer }
$$

where the sum runs over all the massive pairs of fermions and $Q_{1}^{j}+Q_{2}^{j}=p_{j} N$. This equation should be valid for any normalization choice of the massive fields and is a non-trivial constraint on the possible charge assignements of massive fermions. Notice that if the integer in eq. (5) is a multiple of $N$, the massive fermions do not contribute to the cancellation of the discrete anomaly and $r$ in eq.(1) is an integer.

Eq. (5) has implications on the possible charges of massive fermions. To be specific, consider a $Z_{N \times M}$ discrete gauge symmetry such that the massless fermions have charges multiple of $M$. From the low energy point of view only a $Z_{N}$ subgroup will be explicit in the interacting Lagrangian, the massless fermions would have integer $Z_{N}$ charges and the massive fermions will in general have

* Notice that, for $N=3, r$ has to be in fact a multiple of 3 . 
fractional $Z_{N}$ charges proportional to integer $/ M$. The condition (5) will require

$$
\frac{3 N}{M^{2}} \sum_{j} a_{j}=a, a=\text { integer }
$$

where the $a_{j}$ are integer coefficients. It is obvious from this expression that $a M^{2} / 3 N$ has to be an integer. There are two cases now depending whether $a$ is a multiple of $N$ or not. If $a$ is a multiple of $N$, then one can immediately see that $r$ in (1) is an integer also and the anomaly cancellation condition is equal to the one corresponding to integrally charged massive fermions. If $a$ is not a multiple of $N$, then necessarily $M^{2}$ will have to be a multiple of $N$, in order to $a M^{2} / 3 N$ being integer. Thus we are left with two options concerning the cubic $Z_{N}^{3}$ anomaly, either

1) The cubic anomaly condition (1) is verified with $r=$ integer or

2) There are massive fields with fractional $Z_{N}$ charges proportional to $m / M$, $m \in \mathbf{Z}$, with $a M^{2}$ a multiple of $3 N$.

This second case may be, in some cases, quite restrictive. Thus consider a theory in which the massless fermions respect a $Z_{3}$ symmetry $(N=3)$. The minimum value of $M$ for which condition (6) may be verified is $M=3$, meaning that the complete theory has a discrete gauge symmetry as large as $Z_{9}$.

The above shows us how the cubic $Z_{N}^{3}$ anomaly cancellation condition (1) with integer $r$ may be quite informative. Either it is obeyed, or if not, it tells out something about the charges of the massive fermions. On the other hand, there may be cases in which we have extra information about the massive sector (e.g. like some GUTs or string models) and in which the integrality of the charges of the massive fermions may be guaranteed from the beginning.

ii) The mixed $Z_{N^{-}} S U(M)$ anomaly conditions (3) may also be obtained from $S U(M)$ instanton considerations [12]. This may be easily understood from the 't Hooft fermionic operator

$$
O_{t}=\prod_{\text {lights }} \psi \psi \ldots . \psi
$$

where the product extends over all the light fermions with $S U(M)$ quantum 
numbers. The invariance of that operator with respect to the discrete gauge transformation yields the same result as eq. (3) .

This type of low energy Lagrangian interpretation does not seem very immediate in the case of the cubic (1) and gravitational (2) anomaly conditions. The reason for that is clear in the analysis from ref.[5]. The main reason for this difference is that the contribution of the massive fermions with $S U(M)$ quantum numbers to the mixed $Z_{N}-S U(M)$ anomaly vanishes identically, i.e., the anomaly cancels separately both both massless and massive fermions. In fact one should in principle multiply the operator $O_{t}$ by a similar operator $O_{T}$ involving the heavy fermions with $S U(M)$ quantum numbers. Since the discrete anomalies for light and heavy fermions cancel separately this is not necessary in this case.

This is in fact what is different in the other two type of anomalies. In the case of the cubic anomaly the massive fermions may contribute in two manners to the cancellation of the light fields discrete anomalies. 1) the pairs of massive fermions with Dirac masses may contribute if they have fractional $Z_{N}$ charges by providing a fractional $r$ coefficient in eq.(1) ; 2) for even $N$ the massive Majorana fields contribute the last term in eq.(1). In the case of the mixed $Z_{N}$-gravitational anomaly, the contributions of the massive fermions with Dirac masses cancel identically and only the massive Majorana fermions may contribute to the cancellation of the light fields discrete gauge symmetry (the last piece in eq.(2)).

It is obvious from the above discussion of the cancellation of the cubic $Z_{N}^{3}$ and mixed-gravitational discrete gauge anomalies that there is not decoupling of massive fermions in a chiral theory with a massless fermion sector which has discrete gauge anomalies. It is also clear that one cannot understand all the discrete anomaly cancellation conditions merely in terms of instanton physics involving just the massless fermions. Well on the contrary, the mixed $Z_{N^{-}} S U(M)$ anomalies are exceptional in that the massive fermions do not contribute to anomaly cancellation, and that is why such an interpretation is possible. Concerning the mixed gravitational anomaly, in addition to the fact that heavy Majorana particles do not in general decouple from the low-energy physics, there are additional difficulties for an instanton low energy interpretation because of the poor knowlwdge of gravitational instanton physics. An example of these difficulties will be 
shown in the next chapter while discussing the mixed R-symmetry-gravitational anomalies.

The non-decoupling of massive fermions in the presence of discrete gauge anomalies is reminiscent of the work in ref.[13] in which the standard model in the limit of a heavy top quark is considered (more recent related work may be found in ref. [16]). In that case the top quark never really decouples since then one would be left with an anomalous theory. Instead, a Wess-Zumino term is generated which helps to cancel the (perturbative) anomalies. Since a top-less theory would also have a non-perturbative $S U(2)$ anomaly one also adds a sort of 'topological Wess-Zumino' term which helps to cancel that anomaly in the low energy sector. In the case of the standard model, describing the Higgs field $\Phi$ in terms of a unitary matrix $U$ such that $\Phi=\phi U$, this non-perturbative WessZumino term in the action has the form [13]

$$
\Delta S_{1}\left(U, A_{\mu}, B_{\mu}\right)= \begin{cases}0 & \mathrm{U} \text { trivial in } \Pi_{4}(S U(2)) \\ \pi & \mathrm{U} \text { non-trivial in } \Pi_{4}(S U(2))\end{cases}
$$

It is reasonable to conjecture that, since the discrete gauge anomalies here considered are also non-perturbative, the effect of the massive fermions in the low energy theory may also be described by analogous 'topological Wess-Zumino' terms in the action. Consider first the case of the $Z_{N}^{3}$ cubic anomaly. As we discussed, there are two contributions of the massive fermions which help in cancelling the anomaly, one from the massive Dirac pairs and the other from the Majorana massive fields. The contribution of the latter is proportional to $n N^{3} / 8, n \in \mathbf{Z}$ and is only present for even $N$. In fact one can see that this contribution is only non-trivial for $N=2$. Indeed, one can write $N=2 N^{\prime}, N^{\prime} \in$ Z. If $N^{\prime}$ is odd one really has a discrete $Z_{2} \times Z_{N^{\prime}}$ symmetry and it is only the $Z_{2}$ piece which has this term. If $N^{\prime}$ is even, $n N^{3} / 8=n N^{\prime 3}$ is always a multiple of $N=2 N^{\prime}$ and hence vanishes modulo $N$. Thus one only has to worry about this term in a $Z_{2}$ cubic discrete anomaly. Concerning the contribution from the massive Dirac pairs, it will appear through a generation of a non-integer $r_{\psi}$ in eq. (1) (but such that $r_{\psi} N$ is integer), and may be generically present for all $N$. Thus one can achieve cancellation of discrete cubic anomalies from the light 
fermions by formally adding in to the action terms of the form

$$
\Delta S_{\text {cubic }}= \begin{cases}r_{\psi} 2 \pi & \mathrm{N}^{\prime} \text { even } \\ r_{\psi} 2 \pi+n \pi & \mathrm{N}^{\prime} \text { odd }\end{cases}
$$

In the case of the mixed $Z_{N}$-gravitational anomaly, only massive Majorana particles can contribute to the cancellation of the discrete anomaly of the light fermions. One would expect then a term of the form $\Delta S_{\text {grav }}=n^{\prime} \pi$ which would be present only for even $N$ (but for any $N^{\prime} \in \mathbf{Z}$ ).

\section{Discrete R-symmetry Anomalies}

In $N=1$ supersymmetric theories there are two type Abelian internal symmetries: ordinary symmetries and R-symmetries. The first commute with the SUSY generator and the second do not. It is then obvious that one cannot gauge a continuous $U(1)$ R-symmetry since supersymmetry would be broken. On the other hand there do in general exist in certain cases 'discrete gauge Rsymmetries'. As remarked in ref.[6],[12] , if the four-dimensional field theory is obtained from a higher dimensional one (as may be the case in Kaluza-Klein and string theories), there could be R-symmetries which are discrete remnants of the rotation group in the compactified dimensions. Indeed, in explicit 4-dimensional strings like orbifold compactifications those discrete gauge R-symmetries exist. They are 'gauge' in the sense that they originate from the gauge theory of relativity in the extra dimensions.

In addition to that possibility, there is another type of continuous 'gauged R-symmetries' which correspond to $U(1)$ symmetries in supersymmetric sigma models. In this case the gauge connection does not propagate, it is instead an auxiliary vector field which couples in a chiral manner to fermions. This sigma model structure with gauge connections coupling to R-symmetries are in general present in $N=1$ supergravity Lagrangians. They also appear in the particular class of $N=1$ supergravity Lagrangians which correspond to the low energy limit of some four-dimensional strings. In this particular case, these gauged $U(1)$ R-symmetries can be broken down to a $Z_{N}$ R-symmetry subgroup by the vev of some scalar field. 
Using arguments similar to those in ref.[5] one can obtain the equivalent discrete anomaly cancelation conditions for discrete R-symmetries. A generic Rsymmetry associates charges for the gravitino and gaugino fields $Q_{3 / 2}^{R}=Q_{1 / 2}^{R}=1$ and charges for the matter fermions $\psi_{i}$ equal to $q_{i}$. In the process of symmetry breaking $U(1)^{R} \rightarrow Z_{N}$ (while preserving supersymmetry) some matter fermions may get three types of masses: 1) Dirac mass terms combining pairs of matter fields; 2) Majorana mass terms 3) Dirac mass terms combining a matter field with a gaugino field as in the usual supersymmetric version of the Higgs effect. Since, by assumption, supersymmetry is unbroken, there cannot be neither gaugino Majorana mass terms nor mixed gravitino-gaugino nor gravitino-matter fermion terms, since all of these would break supersymmetry either explicitely (in the first case) or spontaneously (in the last two cases).

Let us first consider the mixed discrete R-symmetry-SU(M) anomaly. One can in fact directly apply equation (3) to this case to obtain

$$
\sum_{i} T_{i}\left(q_{i}\right)+M=\frac{1}{2} r^{\prime \prime} N, r^{\prime \prime} \in \mathbf{Z}
$$

where $T_{j}$ is the quadratic $S U(M)$ Casimir corresponding to each given representation and, again, the normalization is such that the Casimir of an M-plet is $=1 / 2$. This expression may be understood [12] by requiring that the corresponding instanton generated operator in eq.(7) vanishes.

The case of the mixed R-symmetry-gravitational anomaly is more complicated since the gravitino field contributes to it. The contribution of spin $3 / 2$ and $1 / 2$ fermions to the usual gravitational chiral anomaly is given by [17]

$$
\int d^{4} x \sqrt{g} D_{\mu} J_{5}^{\mu}=\frac{1}{24}\left\{\frac{1}{16 \pi^{2}} \int d^{4} x \sqrt{(g)} R_{\mu \nu \rho \sigma}^{*} R^{\mu \nu \rho \sigma}\right\}\left(21 N_{3 / 2}-N_{1 / 2}\right)
$$

where $N_{3 / 2}, N_{1 / 2}$ are the number of spin $3 / 2$ and spin $1 / 2$ fermions. This tells us that the contribution of the gravitino is (-21) times what a Weyl spinor contributes. This may be understood counting the number of fermion zero modes in the background of a $K_{3}$ gravitational instanton [18] . Apart from this, one can again use eq.(2) and obtain for the mixed $Z_{N}$-R-symmetry mixed gravitational 
anomaly

$$
\sum_{i} q_{i}-21+\operatorname{dim} G=r^{\prime} N+\eta n^{\prime} \frac{N}{2}, n^{\prime}, r^{\prime} \in \mathbf{Z}
$$

where $\operatorname{dim} G$ is the dimension of the complete gauge group. Notice that there is no obvious way by which the above anomaly cancellation equation could be understood in terms of an effective 't Hooft operator involving just the massless fermions. To start with, such a possible interpretation would not be able to account for the last piece in eq.(12), which represents the contribution of possible massive Majorana fields to the cancellation of the anomaly. Secondly, it is not obvious what fermion operator might account for the factor 21 between gravitino and matter fields. Notice that our eq.(12) does not agree with the result presented in ref.[12] which apparently did not include the correct zero mode counting.

\section{Discrete Gauge Symmetries in String Models and the Discrete} Green-Schwarz Mechanism

In four-dimensional string theories the presence of discrete symmetries is ubiquitous. Many of them are Abelian $Z_{N}$ symmetries which have a gauge character. It is perhaps usefull to enumerate a few examples of discrete gauge symmetries appearing in string models.

i) In four-dimensional strings constructed a la Gepner, the models are obtained by tensoring $N=2$ superconformal minimal models both for right and left movers in such a way that the internal central charge is $c=9$. Each of the superconformal blocks presents a $Z_{k+2}$ symmetry, where $k$ is the level of the minimal model. If there are $\mathrm{n}$ such factors the resulting model has a $\Pi_{i} Z_{k_{i}+2 R} \otimes \Pi_{i} Z_{k_{i}+2}$, $i=1, . . n$ discrete symmetry, although often only a subgroup of this symmetry is realized in the massless particles. Some of these are normal symmetries whereas others are discrete $R$-symmetries. These models also have $n-1 U(1)$ gauge bosons, the so-called enhanced $U(1)$ s. One can easily check in these models the presence of some massless singlet scalars (moduli), which, if given a non-vanishing vev, break the $U(1) \mathrm{s}$ down to some of the $Z_{k_{i}+2}$ symmetries described above. Thus plenty of the discrete symmetries in these models may be understood as discrete gauge symmetries. 
2) Discrete gauge symmetries are also abundant in orbifold four-dimensional strings. For example, the discrete symmetries associated to the $Z_{N}$ or $Z_{N} \times Z_{M}$ point group twists of the six-dimensional tori may be understood as discrete gauge symmetries. Indeed, orbifolds have enhanced $U(1)$ symmetries at some multicritical points of the untwisted moduli (e.g., the $E_{6} \times E_{8} \times S U(3)$ symmetry of the standard $Z_{3}$ orbifold is enhanced by extra $U(1)^{6}$ interactions). The untwisted moduli have integer charges under these enhanced $U(1)$ s whereas the twisted fields have generically fractional charges (i.e., multiple of $1 / N$ for a $Z_{N}$ twist). If the untwisted moduli get non-vanishing vevs, the enhanced $U(1) \mathrm{s}$ are spontaneously broken to $Z_{N}$ subgroups which are then discrete gauge symmetries.

3) The two above examples correspond to 'enhanced' $U(1)$ symmetries breaking down to some abelian discrete gauge group. There are also plenty of examples of gauge groups belonging to the ' $E_{8} \times E_{8}$ ' sector which break also to subgroups containing a gauge $Z_{N}$ subgroup. Let us give here a $Z_{3}$ orbifold example which will also serve us to briefly describe the Green-Schwarz [19] mechanism often at work in four-dimensional strings. Take one of the four modularinvariant $(0,2)$ embeddings of the $Z_{3}$ orbifold, the one leading to the gauge group $E_{7} \times S O(14) \times U(1)_{1} \times U(1)_{A}$. This model may be obtained [20], [21] by embedding the $Z_{3}$ twist in the $E_{8} \times E_{8}$ degrees of freedom through a shift $\vec{V}=1 / 3(110 \ldots 0) \times(20 \ldots 0)$. The massless charged chiral fields of the model are the following: three copies of $(56,1 ; 0,1)+(1,1 ; 0,-2)+(1,14 ;-2,0)+(1,64 ; 1,0)$ in the untwisted sector; 27 copies of $(1,14 ;-2 / 3,2 / 3)+(1,1 ; 4 / 3,-4 / 3)$ in the twisted sector and 81 copies of $(1,1 ; 4 / 3,2 / 3)$ from twisted oscilators. One interesting feature of this model is that the ABJ gauge anomalies of the $U(1)_{A}$ Maxwell field do not cancell, as the reader may easily check. In fact, this is not quite true since the anomaly is cancelled by the four-dimensional version [22] of the Green-Schwarz (GS) mechanism. In a simplified way this mechanism works as follows. In four-dimensional strings the gauge kinetic piece of the Lagrangian has the generic form

$$
\phi(x) \sum_{i} k_{i} F_{i}^{2}+i \eta(x) \sum_{i} k_{i} F_{i} \tilde{F}_{i}
$$

where $\eta(x)$ is the axionic field which is the partner of the string dilaton $\phi(x)$ and 
the sum runs over the different gauge groups of the model. The $k_{i}$ are the KacMoody levels of the different gauge groups. They are integers for non-Abelian groups but may be non-integer numbers for the $U(1)$ s. The GS mechanism works by assigning a non-trivial transformation behaviour of the field $\eta(x)$ under a gauge transformation $V_{A}^{\mu} \rightarrow V_{A}^{\mu}+\partial^{\mu} \theta(x)$ of the anomalous $U(1)_{A}$ :

$$
\eta(x) \rightarrow \eta(x)-\theta(x) \delta_{G S}
$$

where $\delta_{G S}$ is a constant. If the anomaly coefficients $C_{i}$ corresponding to the triangle anomalies involving one $U(1)_{A}$ gauge boson and any other pair of gauge bosons are in the ratio

$$
\frac{C_{1}}{k_{1}}=\frac{C_{2}}{k_{2}}=\ldots . .=\delta_{G S},
$$

the corresponding anomalies will be cancelled by the $U(1)_{A}$ gauge variation of the second term in eq.(13). It is easy to check that in the above $Z_{3}$ orbifold example the anomaly coefficients are in the apropriate ratio and the anomalies are cancelled by this mechanism. One can also see [23] that there is a flat direction in the scalar potential in which one of the $(56,1 ; 0,1)$ massless fields get a vev and break the symmetry:

$$
E_{7} \times U(1)_{A} \rightarrow E_{6} \times Z_{3}{ }^{A},
$$

i.e. the $U(1)_{A}$ symmetry is broken to a $Z_{3}$ gauged subgroup. Under this $Z_{3}{ }^{A}$ symmetry all untwisted fields are singlets whereas all twisted states transform with the phase $\exp (i 4 \pi / 3)$, as the reader may easily check by looking to the $U(1)_{A}$ charges of the fields in the model.

All the above string examples verify the discrete gauge anomaly cancellation conditions. In fact, in the case of the $Z_{3}$ orbifold examples, since the multiplicity of the charged fields is always a multiple of 3 , the anomalies cancel in a trivial way. The same is true for the $Z_{3}{ }^{A}$ example above, even though the original $U(1)_{A}$ is 'anomalous', the residual $Z_{3}{ }^{A}$ is not, due to the multiplicity of the twisted fields. 
In general, if an 'anomalous' $U(1)_{A}$ is broken to a subgroup $Z_{N}{ }^{A}$, the latter will be 'anomalous' only if

$$
\frac{C_{i}}{k_{i}}=\delta_{G S} \neq 0, \bmod N
$$

A model with this property is provided by the $Z_{3}$ orbifold example of ref. [24] - This is a model with three discrete Wilson lines in which the gauge group is $S U(5) \times S U(3) \times S U(2) \times U(1)^{9}$ and one of the $U(1) \mathrm{s}$ is 'anomalous'. The mixed anomalies of this $U(1)$ with the non-Abelian gauge interactions are respectively $C_{5}=C_{3}=C_{2}=-44$ which is $\neq 3 \bmod 3$. The model has a flat direction in the untwisted sector (involving scalar fields corresponding to the same complex plane and with $S U(3) \times S U(2) \times U(1)_{A}$ quantum numbers $(3,2 ;-3),(\overline{3}, 1 ; 0)$ and $(1,2 ;+3))$. When these fields get equal vevs the symmetry breaking $U(1)_{A} \rightarrow Z_{3}$ occurs. This residual $Z_{3}$ is thus apparently 'anomalous' but in fact this anomaly is cancelled by an apropriate discrete shift of the axion field $\eta(x)$. This 'discrete Green-Schwarz mechanism', as its continuous counterpart, is likely to be present in many specific four-dimensional strings. Another example in a Gepner-type construction with Wilson lines was given in ref. [12].

\section{Phenomenological Applications}

Discrete symmetries are often used in order to constrain Lagrangians for a variety of phenomenological problems. An important question is whether there is any reason why low energy discrete symmetries should be gauged. I can think of three main motivations for that assumption. 1) Gauge discrete symmetries are stable against gravitational (e.g., wormholes) corrections which have been argued violate all global symmetries. 2) Most (may be all) discrete symmetries of the effective Lagrangian in 4-dimensional strings may be understood as gauge discrete symmetries. 3) The usual theoretical prejudice in favour of local versus global symmetries. Assuming that the low energy discrete symmetries come somehow from a larger gauge symmetry gives a rationale for the very existence of discrete symmetries. On the contrary, the existence of discrete global symmetries seem unmotivated from a fundamental point of view. 
If one accepts that low energy discrete symmetries are gauge, they must obey the anomaly cancellation conditions discussed in chapter 2 . These equations then constrain the possible allowed discrete symmetries in a substantial manner. It makes then sense to check what are the discrete symmetries used in the most popular phenomenological Lagrangians and check for discrete gauge anomaly cancellation conditions. Examples of relevant discrete symmetries are the following:

1) Discrete symmetries supressing flavour changing neutral currents in twoHiggs models.

These are needed in generic extensions of the standard model involving more than one Higgs doublet. Consider the two Higgs-doublet case and a generationblind $Z_{N}$ symmetry acting on the five independent standard model fermion multiplets $Q, u, d, L, e$ and the two Higgs doublets with opposite hypercharges $H, \bar{H}$ as

$$
\Psi_{j} \rightarrow e^{i \alpha_{j} 2 \pi / N} \Psi_{j} \quad j=Q, u, d, L, e, H, \bar{H}
$$

where the $\alpha_{j}$ are integers. The absence of flavour-changing neutral currents (FCNC) is guaranteed if the Higgs which couples to the charge $2 / 3$ quarks is different from the one which couples to the charge $1 / 3$ quarks and the leptons [25] . Consider the three independent $Z_{N}$ generators discussed in ref.[6]

$$
R_{N}=e^{i 2 \pi I_{3}^{R} / N}, A_{N}=e^{i 2 \pi Y_{A} / N}, L_{N}^{j}=e^{i 2 \pi L^{j} / N}
$$

where $j=1,2,3$ correspond to the three lepton numbers ${ }^{*}$ The form of the charge generators $I_{3}^{R}, Y_{A}, L^{j}$ is shown in table 1 . The invariance of the standard model Lagrangian with respect to $I_{3}^{R}$ and $L^{j}$ correspond to the invariance of the renormalizable Lagrangian with respect to Baryon and Lepton numbers. If one adds a second Higgs doublet, one has to imposse some symmetry in order to ensure the absence of FCNC, as we remarked above. It is easy to check that the

* We will later on denote the equivalent overall lepton number generator by $L$. 
most general discrete symmetry which works may be written as

$$
S_{N}=R_{N}^{m} \times\left(L_{N}^{j}\right)^{p_{j}} \times A_{N}
$$

i.e., essentially the discrete $A_{N}$ generator, up to discrete baryon or lepton number discrete generators.

Following the above argumentation, one should imposse that the $S_{N}$ symmetry should be anomaly-free. The mixed $S_{N^{-}} S U(3)$, and $S U(2)$ anomaly coefficients are given by [26]

$$
\begin{aligned}
C_{3} & =-\frac{N_{g}}{2} \\
C_{2} & =-\frac{N_{g}}{2}-\frac{\sum_{j}^{N_{g}} p_{j}}{2}
\end{aligned}
$$

where $N_{g}$ is the number of quark-lepton generations. The coefficients $C_{3}, C_{2}$ should vanish modulo $N / 2$ for those anomalies to cancel, and this only happens for $N=N g=3$. Thus the unique anomaly free $S_{N}$ symmetry guaranteeing absence of flavour changing neutral currents is $A_{3}$. The other possibilities are, either anomalous or related to $A_{3}$ by discrete baryon or lepton number rotations. Notice that the mixed $A_{3}$-gravitational anomaly yields $C_{g r a v}=N_{g}(-3-2)=$ $-15=0 \bmod 3$ and hence it also vanishes.

If one allows for the existence of a discrete Green-Schwarz mechanism along the lines of the previous sections, new possibilities appear. In fact, as shown in ref.[26] , in this case a continuous $U(1)_{A}$ symmetry coupling to the $Y_{A}$ generator in table 1 may be gauged and its mixed anomalies with the standard model interactions may be cancelled through a continuous Green-Schwarz mechanism. Correspondingly, discrete symmetries $A_{N}$, for any $N$, may be gauged if the corresponding mixed anomalies are cancelled by a discrete Green-Schwarz mechanism. For example, looking at eqs.(20) one sees that the condition $C_{3}=C_{2}, \bmod N / 2$ for discrete GS mechanism to work yields the condition $\sum_{j}^{N_{g}} p_{j}=0 \bmod N$ and no constraint on $m$. (Here we have assumed the standard values $k_{3}=k_{2}$. Using different k's would allow for even more possibilities). We thus conclude that, in the absence of a discrete GS mechanism, $S_{3}$ symmetries are the unique anomalyfree symmetries supressing FCNC. In the presence of a discrete GS mechanism, 
all discrete $S_{N}$ symmetries in eq.(19) satisfying $\sum^{j} p_{j}=0 \bmod N$ may in principle be gauged. Notice also that the number of possibilities may be further restricted e.g., if one also imposses cancellation of mixed gravitational anomalies. Of course, this type of analysis may be easily extended to models with a more complicated Higgs structure.

In ref.[15] an anomalous global $Z_{2}$ symmetry corresponding to a $S_{2}$ generator was considered. It is clear from the above discussion that this symmetry may be made anomaly free (and hence be gauged) if one includes a discrete GS mechanism as above.

2) Generalized Matter Parities in the Supersymmetric Standard Model

A general analysis of the possible generation-independent discrete gauge symmetries ensuring proton stability in the supersymmetric standard model (SSM) was presented in ref.[6]. The result of the analysis shows that, assuming the particle content of the SSM, there are only a few discrete $Z_{N}$ generalized matter parities which are anomaly free. In terms of the generators in eq.(18) one can write the flavour-independent discrete symmetries as [6]

$$
g_{N}=R_{N}^{m} \times A_{N}^{n} \times L_{N}^{p}
$$

The finite list of such anomaly free symmetries is as follows:

i) A $Z_{3}$ called 'baryon parity' $B_{3}$ which is generated by $B_{3}=R_{3} L_{3}$. The phase assignements of matter fields with respect to this symmetry are $g_{(Q, u, d, L, e)}=$ $\left(1, \alpha^{2}, \alpha, \alpha^{2}, \alpha^{2}\right)$, where $\alpha=\exp (i 2 \pi / 3)$. This is the only symmetry which is anomaly-free just with the massless fermion content, without the need to include a possible effect from massive fermions. It also forbids the presence of dangerous dimension five operators which may give rise to fast proton decay.

ii) The standard $Z_{2}$ R-parity. This is equivalent to the symmetry generated by $R_{2}$. In this case the mixed gravitational anomaly only cancels if there are massive Majorana fermions (behaving like right-handed neutrinos) which help to cancel the anomaly (last piece in eq.(1)).

iii) There are three other $Z_{3}$ symmetries which can be made anomaly-free if there are massive fermions with fractional $Z_{3}$ charges. They are the symmetries 
generated by $R_{3}, L_{3}$ and $R_{3} L_{3}^{2}$. These symmetries seem to obey all the anomalycancellation conditions (1) -(3) . However, as indicated in the footnote in section 2, for $Z_{3}$ symmetries $r$ must be a multiple of 3 (and $r N$ a multiple of 9 ) if the massive particles have integer $Z_{3}$ charges. This is the case for $B_{3}$, but not for these other three symmetries. This implies that, for these three symmetries to be anomaly-free, there must be massive fractionally charged fermions helping in cancelling the anomaly. As discussed in section 2, this means that the actual symmetry of the theory is at least as large as $Z_{9}$ and not just merely $Z_{3}$.

This is the finite list of anomaly free possibilities for the particle content of the minimal SSM. The three symmetries in iii) were overlooked in the preprint version of ref.[6] and included in the published version. Although less atractive than $B_{3}$ and $R_{2}$ because they involve larger order symmetries, they cannot be discarded merely on anomaly cancellation grounds.

If we enlarge the spectrum by considering an axion-dilaton system making possible a discrete version of the GS mechanism, the number of potentially anomaly-free solutions substantially increases. Let us consider just the mixed $Z_{N^{-}} S U(3)$ and $S U(2)$ anomalies for the moment. For a discrete GS mechanism to work one needs to have (see eq.(20))

$$
\begin{aligned}
-n N_{g} & =2 \delta_{G S} k_{3} \\
-n N_{g}-p N_{g}+n N_{D} & =2 \delta_{G S} k_{2} \quad, \bmod N
\end{aligned}
$$

where $N_{g}$ is the number of generations and $N_{D}$ the number of Higgs pairs. $\delta_{G S}$ is the Green-Schwarz term coefficient which is a model dependent constant and $k_{2}, k_{3}$ are the levels of the groups. Normally one assumes $k_{2}=k_{3}$ (it is required in order to obtain reasonable coupling constant unification predictions) and then, substituting $\delta_{G S}$ one gets after some trivial algebra

$$
p-n \frac{N_{D}}{N_{g}}+t \frac{N}{N_{g}}=0 \quad, \quad t \in \mathbf{Z}
$$

and again no constraint on $m$ (the $I_{3}^{R}$ generator has no mixed $S U(2), S U(3)$ anomalies). It is easy to find solutions to these equations: The $Z_{2}$ symmetries generated by $A_{2} L_{2}(m=0, n=p=1)$ and $R_{2} A_{2} L_{2}(m=n=p=1)$ can be 
made anomaly-free through this mechanism. For $Z_{3}$ symmetries one has solutions only for $n=0 \bmod 3$ and hence no new symmetries can be made anomaly-free through the GS mechanism. On the other hand, and unlike the case without GS mechanism, $Z_{N}$ symmetries with $N>3$ may be made anomaly free. Examples are the $Z_{4}$ symmetry generated by $A_{4}^{-1} L_{4}$ and the $Z_{5}$ symmetry generated by $A_{5} L_{5}^{2}$.

In addition to eq.(22) one can further imposse cancellation of mixed gravitational anomalies. The equivalent condition is now

$$
-(5 n+p-m) N_{g}+2 n N_{D}+\frac{1}{2} \eta s N=24 \delta_{G S} \bmod N, s \in \mathbf{Z}
$$

where $\eta=1,0$ for $N=$ even, odd. The factor 24 accounts for the graviton normalization and plays the same role as the $k_{i}$ do for gauge fields. Identifying the $\delta_{G S}$ coefficient with those in eq.(22)yields further constraints. In particular, the two symmetries $A_{2} L_{2}$ and $R_{2} A_{2} L_{2}$ are anomaly free whereas the above $Z_{5}$ example is not.

We thus observe that the existence of a dilaton-axion multiplet with transformation properties as required by the discrete GS mechanism increases the number of possibilities. However, since the natural setting for the existence of such a mechanism is string theory, one might consider any evidence in favour of any of these new symmetries as indirect evidence for string theory.

One point not discussed in ref.[6] was the anomaly cancellation constraints on the generalized $R$-matter parities, i.e., discrete R-symmetries protecting the SSM from too fast proton decay. Since string and other higher dimensional theories R-symmetries may also be gauge in origin, it is worth checking how effective are the anomaly cancellation conditions for these type of R-symmetries. The most general discrete R-symmetry of the SSM can be writen as

$$
\tilde{g}_{N}=\tilde{P}_{N} \times R_{N}^{m} \times A_{N}^{n} \times \prod_{j} L_{N}^{j p_{j}}
$$

where $j$ runs over lepton numbers and $\tilde{P}_{N}$ is defined by $\tilde{P}_{N}(d \theta)=\exp (i 2 \pi / N)$ 
$(d \theta), \theta$ being the superspace Grassman variable ${ }^{*}$. On the other hand, the action on the Higgs fields $H, \bar{H}$ is defined in a slightly different way so that the usual Yukawa couplings are always allowed (see table 1) Let us consider to start with the mixed R-symmetry-SU(3) and $S U(2)$ anomalies from a generic generationindependent R-symmetry

$$
\begin{aligned}
6-4 N_{g}-n N_{g} & =0 \bmod N \\
4-4 N_{g}-(n+p) N_{g}+N_{D}(2+n) & =0 \bmod N
\end{aligned}
$$

For the case of the minimal SSM one has $N_{g}=3, N_{D}=1$ and those two equations reduce to $3 p-n=0$ and $6+3 n=0 \bmod \mathrm{N}$, with arbitrary $m$.

There is no non-trivial $Z_{2}$ generalized matter R-parity which is anomaly free ( $\tilde{P}_{2}$ is trivial since it corresponds to a $Z_{2}$ fermion number). On the other hand, one-third of the $Z_{3}$ R-symmetries (eight of them) are anomaly-free. We list them here for convenience of the reader: $\tilde{P}_{3}, \tilde{P}_{3} R_{3}, \tilde{P}_{3} L_{3}, \tilde{P}_{3} L_{3}^{2}, \tilde{P}_{3} L_{3} R_{3}, \tilde{P}_{3} L_{3} R_{3}^{2}$, $\tilde{P}_{3} L_{3}^{2} R_{3}, \tilde{P}_{3} L_{3}^{2} R_{3}^{2}$. One can further impose the cancellation of mixed gravitational anomalies eq.(12) which yields

$$
-21+12+N_{g}(-15-5 n-p+m)+N_{D}(4+2 n)=0 \bmod 3 .
$$

This equation has no solution for the realistic case with $N_{g}=3, N_{D}=1$. Thus, unless one adds extra singlets to the minimal SSM, there are no anomaly free $Z_{3}$ R-symmetries stabilizing the proton. On the other hand, extra singlets are likely to exist anyhow in supergravity theories so that one should not rule out completely the above symmetries. Unlike the case of standard discrete generalized matter parities, there are also plenty of solutions for $N$ higher than 3 . Thus, e.g., the $Z_{4}$ symmetry $\tilde{P}_{4} L_{4}^{2} A_{4}^{2}$ has no mixed $S U(2)$ and $S U(3)$ anomalies. On the other hand the $\tilde{P}_{4}$ symmetry (equivalent to the $P_{2}$ of ref.[6]) is anomalous. The number of possibilities may be reduced by impossing also the cancellation of mixed R-symmetry-gravitational anomalies as in the $Z_{3}$ cases above. This I

* Notice that the definition of $\tilde{P}_{N}$ here is different from that of $P_{N}$ in ref.[6] by a factor of 2 in the exponent. This is done so that the action on the fermions is generically a $Z_{N}$ and not a $Z_{2 N}$ symmetry. Note e.g. that the $P_{2}$ symmetry in ref.[6] is in fact a $Z_{4}$ symmetry whereas $\tilde{P}_{2}$ here is a genuine $Z_{2}$. 
leave as an exercise to the reader. Additional anomaly-free R-symmetries may be found if one allows for cancellation of anomalies through a discrete GS mechanism involving the R-symmetries.

As a general conclusion one sees that the possibilities for anomaly-free symmetries and R-symmetries stabilizing the proton are increased if 1) one allows for a discrete GS mechanism and 2) one allows for gauged R-symmetries. For $N<4$, however, only a couple of new $Z_{2}$ symmetries, which correspond to $A_{2} L_{2}$ (a 'lepton parity') and $R_{2} A_{2} L_{2}$ (a 'baryon parity') may be made anomaly free (a discrete GS mechanism is required). A discrete GS mechanism would also be required to get new anomaly free R-symmetries for $N<4$.

The above new anomaly free symmetries are only really expected in theories with higher dimensions like strings. In some way, any evidence for this type of symmetries would be an indirect evidence for higher dimensions. In theories without higher dimensions only the few $Z_{2}, Z_{3}$ symmetries described at the begining of this section are anomaly free. Out of those only the baryon-parity $B_{3}$ is anomaly-free without the help of contributions from hypothetical heavy fermions.

\section{Final Comments}

In the approach described here the cancellation of discrete gauge symmetries is used as a constraint on low energy physics. A different approach has been considered in ref.[15] and [27]. These authors consider anomalous (but, in general, non-gauge) discrete symmetries. If these discrete symmetries are anomalous, the QCD anomaly may give rise to interesting effects which could be of use in solving the domain-wall problem present in some models. This is an interesting scenario which could be present e.g. in many grand unified theories. However, as I argued above, it is reasonable to assume that the discrete symmetries are gauge in order to protect the symmetries from large gravitational effects. Furthermore, a gauge origin for discrete symmetries gives a rationale for their very existence. String theories, as I described above, have plenty of discrete gauge symmetries and all the examples found up to now are anomaly free. This is why I believe impossing the constraint of anomaly cancellation is an interesting constraint. This seems an approach orthogonal to that of ref.[15] and [27] which make use of anomalous discrete symmetries, a situation not expected in string models. 
In the case in which a discrete GS mechanism is at work, if one ignores the axion-dilaton sector, the rest of the massless fermionic sector looks anomalous, although the overall theory is anomaly-free. It is in this sense [12] that one can say that anomalous discrete symmetries may appear in string models. However, it is not clear that one can use the arguments of [15] for this kind of 'anomalous' discrete symmetries since the effects of the dilaton-axion sector cannot in general be neglected at high temperatures. Furthermore, the discrete symmetry will also necessarily have mixed $S U(2)_{L}$ anomalies whose non-perturbative effects at finite temperature also have to be included. All these points would have to be taken into account in trying to use the philosophy of ref.[15] to these 'pseudoanomalous' discrete symmetries appearing in some string models with a discrete GS mechanism. 


\section{REFERENCES}

[1] For a review and references see: T. Banks, Physicalia Magazine 12 (1990) 19.

[2] L. Krauss and F. Wilczek, Phys.Rev.Lett. 62 (1989) 1221.

[3] T. Banks, Nucl. Phys. B323 (1989) 90;

L. Krauss, Gen.Rel.Grav. 22 (1990) 50;

M. Alford, J. March-Russell and F. Wilczek, Nucl. Phys. B337 (1990) 695;

J. Preskill and L. Krauss, Nucl. Phys. B341 (1990) 50;

M. Alford, S. Coleman and J. March-Russell, Nucl. Phys. B351 (1991) 735.

[4] J. Preskill, Ann.Phys. 210 (1991) 323.

[5] L.E. Ibáñez and G.G. Ross, Phys. Lett. $\underline{260 B}$ (1991) 291.

[6] L.E. Ibáñez and G.G. Ross, Nucl. Phys. B368 (1992) 3.

[7] D. Kapetanakis, P. Mayr and H.P. Nilles, Phys. Lett. $\underline{282 B}$ (1992) 95.

[8] E. Chun and A. Lukas, Munich preprint TUM-TH-150/92 (1992).

[9] S.P. Martin, Gainesville preprint UFIFT-HEP (1992).

[10] P.L. White, Southampton preprint SHEP-91/92-24 (1992).

[11] K.W. Choi, D. Kaplan and A. Nelson, preprint UCSD-PTH-92-11 (1992);

M. Dine, R. Leigh and D. MacIntire, preprint SCIPP-92-16 (1992).

[12] T. Banks and M. Dine, Phys.Rev.D45 (1992) 1424.

[13] E. D'Hooker and E. Farhi, Nucl. Phys. B248 (1984) 59.

[14] S.-J. Rey, Yale preprint YCTP-P14-92 (1992).

[15] J. Preskill, J. Trivedi, F. Wilczek and M. Wise, Nucl. Phys. B363 (1991) 207.

[16] T. Banks and A. Dabholkar, Rutgers preprint RU-99-09 (1992).

[17] P. van Nieuwenhuizen, Phys.Rep. 68 (1981) 191.

[18] T. Eguchi, P. Gilkey and A. Hanson, Phys.Rep. 66 (1980) 214.

[19] M. Green and J. Schwarz, Phys. Lett. $\underline{149 B}$ (1984) 117. 
[20] L. Dixon, J. Harvey, C. Vafa and E. Witten, Nucl. Phys. B274 (1986) 285.

[21] L.E. Ibáñez, H.P. Nilles and F. Quevedo, Phys. Lett. $187 B$ (1987) 25.

[22] E. Witten, Phys. Lett. $149 B$ (1984) 351;

W. Lerche, B. Nilsson and A.N. Schellekens, Nucl. Phys. B299 (1988) 91;

M. Dine, N. Seiberg and E. Witten, Nucl. Phys. B289 (1987) 585;

J. Atick, L. Dixon and A. Sen, Nucl. Phys. B292 (1987) 109.

[23] A. Font, L.E. Ibáñez, H.P. Nilles and F. Quevedo, Nucl. Phys. B307 (1988) 109.

[24] E.J. Chun, J.E. Kim and H.P. Nilles, Nucl. Phys. B370 (1992) 105.

[25] S.L. Glashow and S. Weinberg, Phys.Rev.D15 (1977) 1958.

[26] L.E. Ibáñez, CERN-TH.6501./92 (1992).

[27] G. Lazarides and Q. Shafi, preprint BA-92-32 (1992);

L. Krauss and S.J. Rey, preprint YCTP-P9-92 (1992). 


\begin{tabular}{|c|c|c|c|c|c|c|c|c|}
\hline & $Q$ & $u$ & $d$ & $L$ & $e$ & $\tilde{H}$ & $\tilde{H}$ & $\tilde{g}, \tilde{W}, \tilde{B}$ \\
\hline$R$ & 0 & -1 & 1 & 0 & 1 & -1 & 1 & 0 \\
\hline$Y_{A}$ & 0 & 0 & -1 & -1 & 0 & 1 & 0 & 0 \\
\hline$L_{i}$ & 0 & 0 & 0 & -1 & 1 & 0 & 0 & 0 \\
\hline$\tilde{P}$ & -1 & -1 & -1 & -1 & -1 & 1 & 1 & 1 \\
\hline
\end{tabular}

Table 1. Generators of symmetries in the SM and SSM 